\title{
Views from Understanding Evolution: Parsimonious Explanations for Punctuated Patterns
}

\author{
Anastasia Thanukos
}

Published online: 19 January 2008

(C) Springer Science + Business Media, LLC 2007

Keywords Punctuated equilibria - Allopatric speciation . Peripatric speciation · Parsimony $\cdot$ Teaching

Antievolution groups have frequently pointed to the debate prompted by Niles Eldredge and Stephen Gould's (1972) proposal of punctuated equilibria with triumph. "Look," they've claimed, "biologists can't even agree among themselves how evolution works. The theory must be failing." In the article included in this issue, "Editor's Corner: The Early "Evolution" of "Punctuated Equilibria"," Niles Eldredge (2008) takes on that mischaracterization. He describes the roots of punctuated equilibria and reveals how it builds on (not tears down!) established evolutionary theory.

The idea of punctuated equilibria was inspired by a mysterious pattern in the fossil record. Many fossil organisms have retained the same anatomy for millions of years, appearing to evolve in body form hardly at all-a phenomenon that Eldredge and Gould termed stasis. Then, suddenly, the organisms seem to have experienced a jolt of evolution, quickly evolving into related, but new, species (Fig. 1a,b). Paleontologists see this pattern in many (although far from all) fossil organisms-from coral-like marine bryozoans to large terrestrial mammals, like horses (Gould and Eldredge 1993).

Such observations are at odds with the pattern we might typically imagine evolution generating: slow and steady

A. Thanukos $(\bowtie)$

Museum of Paleontology, University of California,

1101 Valley Life Sciences Building,

Berkeley, CA 94720-4780, USA

e-mail: thanukos@berkeley.edu

URL: http://evolution.berkeley.edu change (Fig. 1c) - in which, for example, tracing fossils through rock layers that correspond to a slow cooling in Earth's climate reveals the gradual evolution of traits suited to colder temperatures. This picture of slow evolution, which Eldredge and Gould termed phyletic gradualism, fits well with one of the keystones of evolutionary theory: natural selection, the process responsible for adapting populations to changes in their environments. It is easy to imagine natural selection, for example, slowly transforming a delicate tropical species into a hardy, well-insulated, coldtolerant species over many millions of years. In some cases, we do observe such gradualistic patterns in the fossil record, but in many others, we observe evolution in apparent fits and starts. If the evolutionary mechanism of natural selection helps explain apparently gradual evolution, what mechanism can help explain punctuated patterns? Do we need to throw out everything we know about how evolution works or search for an as-yet-undiscovered evolutionary process? No.

The beauty of punctuated equilibria is that the idea explains the apparent jolts of evolution seen in the fossil record with evolutionary processes that we already know to be at work in the world: speciation, migration, natural selection, and genetic drift. Eldredge and Gould simply showed how these mechanisms could work together to produce punctuated patterns in fossils. To see exactly how their idea works, it will help to review two modes of speciation: allopatric and peripatric speciation.

\section{Allopatric Speciation}

Allopatric speciation is just a fancy name for speciation by geographic isolation $($ allo $=$ other, patric $=$ place $)$. In this 
Fig. 1 Hypothetical stratigraphic records containing fossil snail shells. (a) A punctuated pattern in which one species appears to have quickly evolved into a new species. (b) A punctuated pattern in which one lineage appears to have quickly produced two daughter species, one with a form identical to the ancestor and one with a distinct form. (c) A pattern recording slow and steady evolutionary change

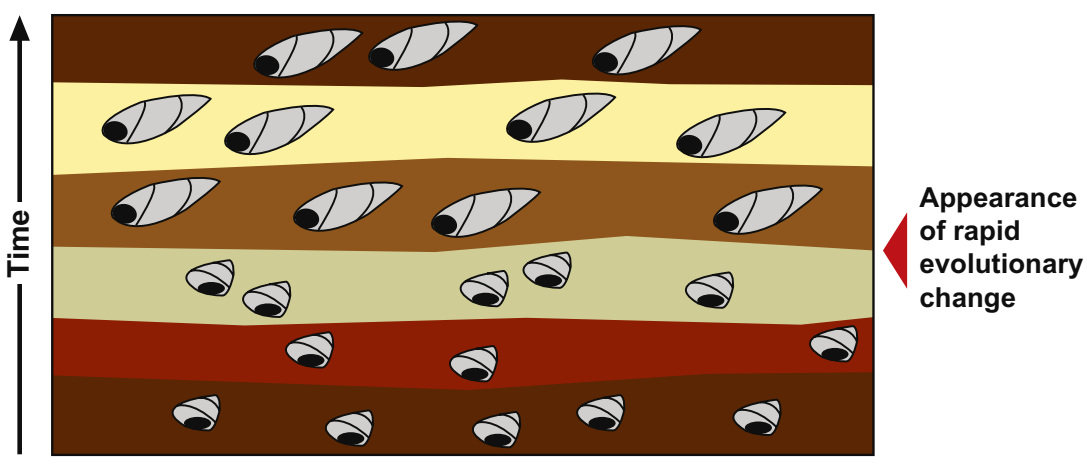

a

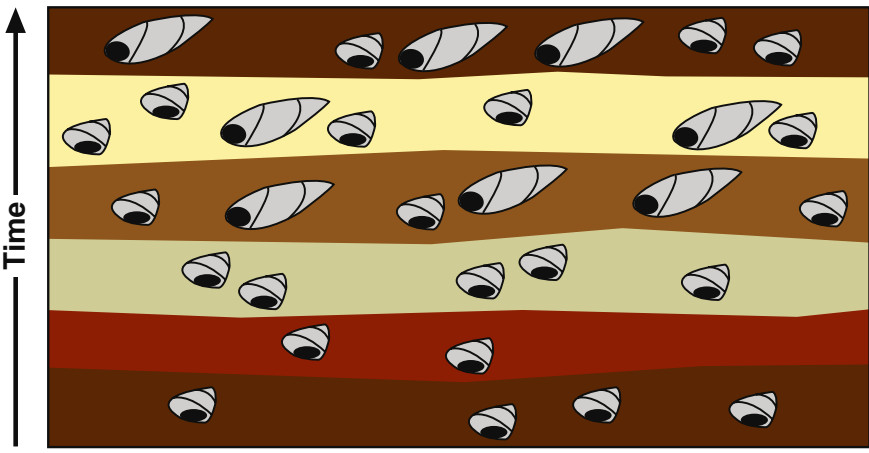

b

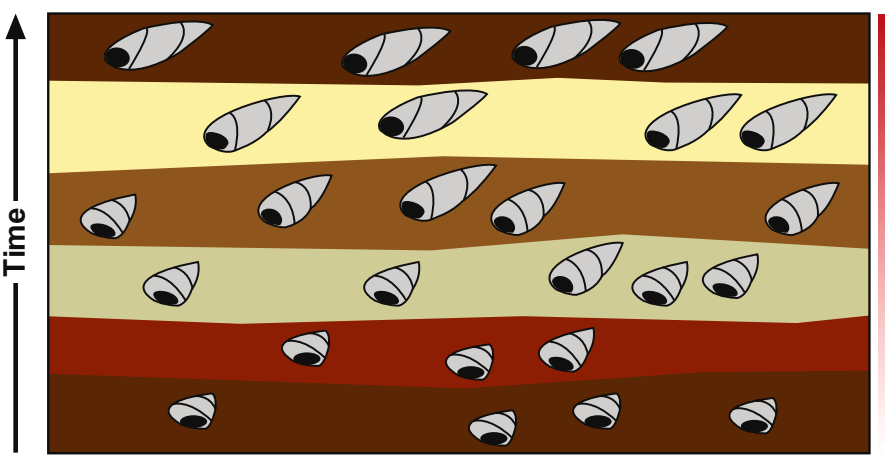

Gradual evolutionary change recorded in fossils process, a geographic barrier divides a population and prevents two or more groups from mating with each other regularly, eventually causing that lineage to speciate. Scientists think that geographic isolation is a common way for the process of speciation to begin: rivers change course (as in Fig. 2), mountains rise, continents drift, organisms migrate, and what was once a continuous population is divided into smaller populations. However the division happens, eventually the subpopulations evolve genetic differences (perhaps triggered by the different

Fig. 2 Allopatric speciation. A beetle population is divided in two by a river. Over time, each subpopulation evolves genetic differences. They eventually evolve into distinct species
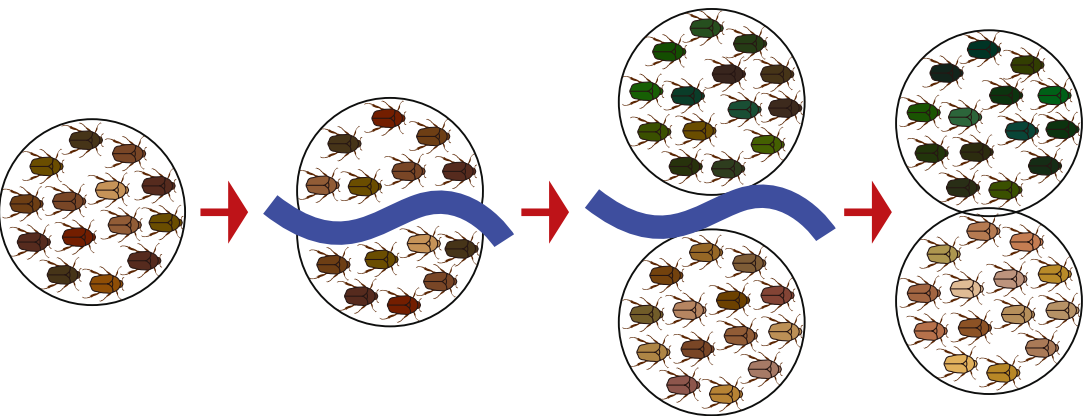
selective pressures in their separate environments) and become discrete species, unable to mate with one another and reunite their genes.

As an illustration of how this process might work, consider the following scenario from the Understanding Evolution website (illustrated in Fig. 3):

1. The scene: a population of wild fruit flies is minding its own business on several bunches of rotting bananas, cheerfully laying their eggs in the mushy fruit, when...

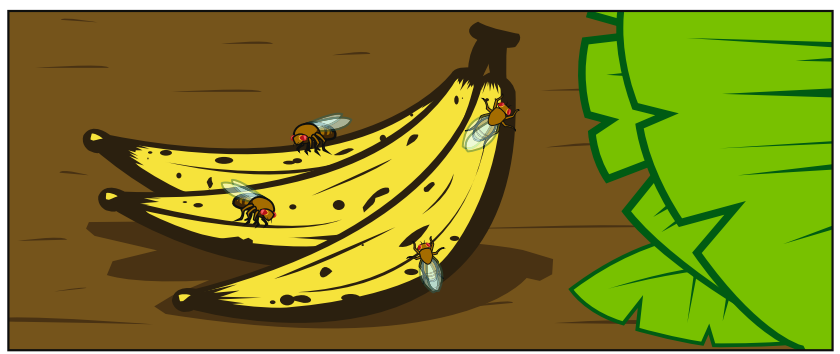

The scene

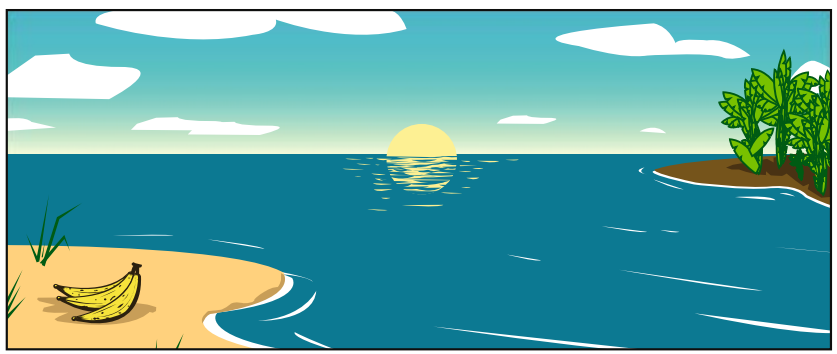

Disaster strikes

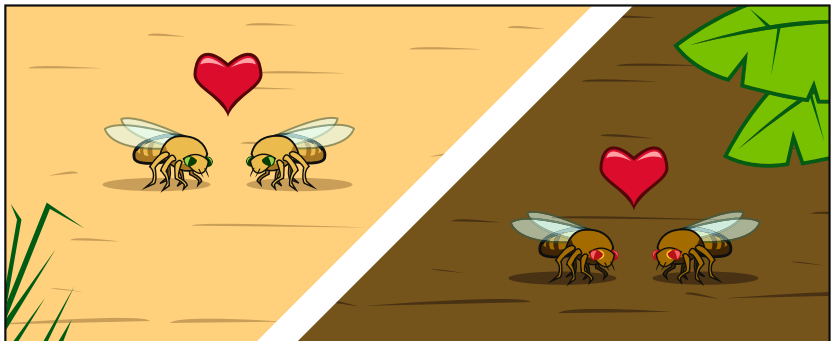

The populations diverge

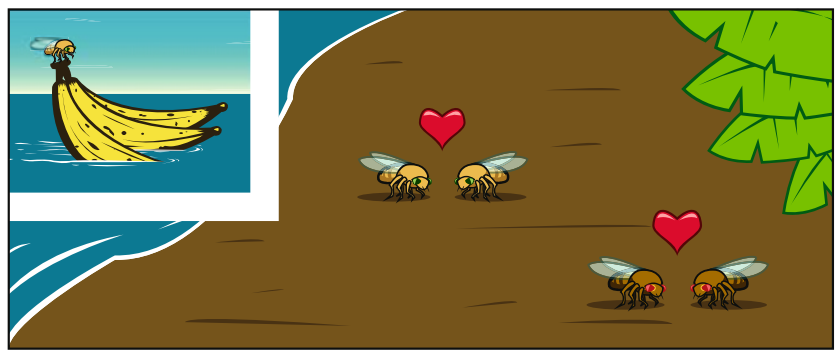

So we meet again

Fig. 3 A hypothetical example of allopatric speciation. Fruit flies from a mainland population are carried to an island in a hurricane. They evolve in isolation on the island and evolve genetic differences from the mainland population. Eventually, the island population evolves into a distinct species, which no longer mates with members of the mainland species
2. Disaster strikes and the population is divided: A hurricane washes the bananas (and the fruit fly larvae they contain) out to sea. The banana bunch eventually washes up on an island off the coast of the mainland. The fruit flies mature and emerge from their slimy nursery onto the lonely island. The two portions of the population, mainland and island, are now too far apart for gene flow to unite them. At this point, speciation has not occurred: any fruit flies that got back to the mainland could mate and produce healthy offspring with the mainland flies.

3. The populations diverge: Ecological conditions are slightly different on the island, and the island population evolves under different selective pressures and experiences different random events than does the mainland population. Body form, food preferences, and courtship displays change over many generations of natural selection.

4. So we meet again: When another storm reintroduces the island flies to the mainland, they will not readily mate with the mainland flies because they've evolved different courtship behaviors. The few that do mate with the mainland flies produce inviable eggs because of other genetic differences. The lineage has split now that genes cannot flow between the populations.

In the example above, the fruit flies alone were carried to an isolated location where speciation occurred. But, of course, when geographic isolation is caused by mountain ranges rising or by climate change that fragments a habitat into favorable and unfavorable patches, more than one species is likely to be affected. In fact, many different species living in the same area may simultaneously experience allopatric speciation if they are all similarly affected by large-scale physical disturbances.

\section{Peripatric Speciation}

Now consider how the scenario described above might play out if one of the populations were unusually small. At first, it might seem that the difference is of no consequence, but in fact, this brings an additional mechanism of evolution into play. Genetic drift-random sampling error in populations - has a disproportionate impact on small populations, and so may play an important role in the divergence of the subpopulations when one of those subpopulations is small. This special case of allopatric speciation is called peripatric speciation $($ peri $=$ near, patric $=$ place $)$. To see how it works, we will return to our intrepid fruit flies venturing off the mainland on a bunch of rotting bananas. 
We pick up their story as their banana bunch washes up on an island (illustrated in Fig. 4):

1. Double disaster: Not only are the island fruit flies now geographically isolated from their mainland relatives, but only a few larvae have survived the harrowing journey to colonize the island.

2. Rare genes survive: These few survivors, by chance, carry some genes that are rare in the mainland population. One of these rare genes happens to cause a slight variation in the mating dance. Another causes a slight difference in the shape of the male genitalia. This is an example of the founder effect - a change in gene frequency that occurs because the genes in a newly founded population do not happen to be representative of those in the source population.

3. Gene frequencies drift: Although rare on the mainland, these small differences become common in the island population over the course of a few generations through the action of genetic drift. In fact, the entire island population ends up carrying these genes.

4. More changes: As the island population grows, the unique reproductive features on the island result in a cascade of changes caused by sexual selection-for example, improved fit of male and female genitalia to one another and increased female responsiveness to the mating dance. Flies also experience natural selection that favors individuals better suited to the climate and food of the island.

5. Speciation: After some generations, the island flies become reproductively isolated from the mainland flies. Peripatric speciation has occurred.

Because genetic drift acts more quickly in small populations, full-blown speciation is a more likely result of geographic isolation when one of the isolated populations is small. In this situation, genetic drift, and perhaps strong selective pressure, can cause rapid genetic change in the small population.

\section{Putting the Pieces Together}

Eldredge and Gould's proposal of punctuated equilibria was inspired by imagining what patterns in the fossil record

Fig. 4 A hypothetical example of peripatric speciation. Just a few fruit flies from a mainland population are carried to an island in a hurricane. These flies happen to have an unusually high frequency of two genes that are rare in the mainland population. Those rare genes happen to drift to fixation in the island population. Over time, this precipitates other evolutionary changes in the island population, and they evolve into a separate species from the mainland flies

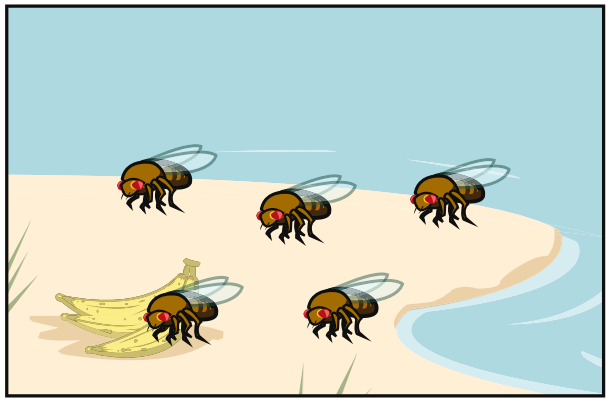

Double disaster

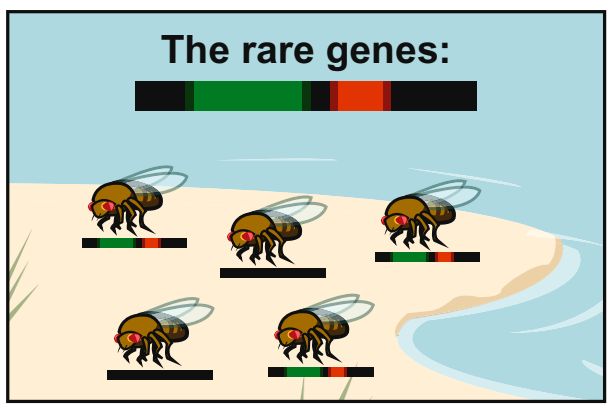

Rare genes survive

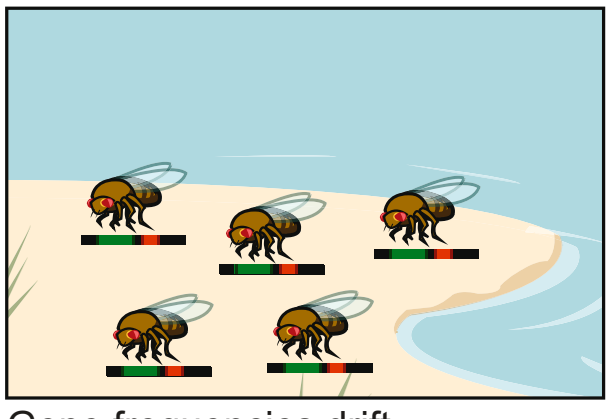

Gene frequencies drift

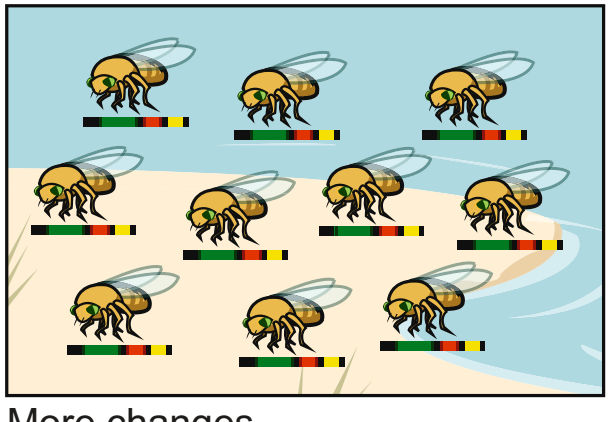

More changes

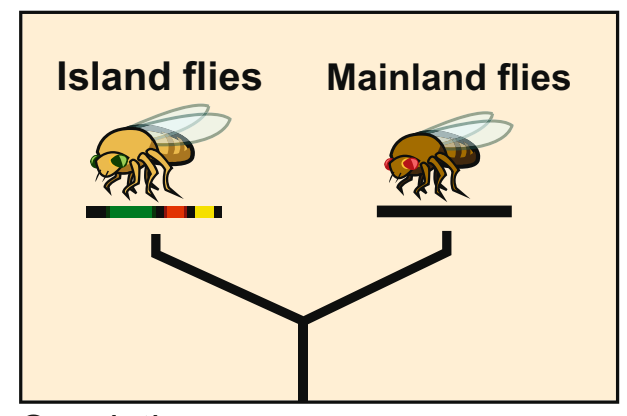

Speciation 
might be produced by allopatric, and specifically peripatric, speciation. Fossilization is a spotty event-both historically and geographically. Over a million years or more, fossils might be preserved from only a few time periods. Similarly, over a wide area, fossils might be preserved in only a few places. Given these constraints, let us take a look at what fossils a snail lineage undergoing peripatric speciation might leave behind (illustrated in Fig. 5):

1. Stasis: A population of snails is experiencing stasis, living, dying, and getting fossilized every few hundred thousand years. Judging from these fossils, little evolution seems to be occurring.

2. Isolation: A drop in sea level forms a lake and isolates a small number of snails from the rest of the population. This is the first step in peripatric speciation.

3. Strong selection and rapid change: The environment in the newly formed lake exerts new selection pressures on the isolated snails. Also, their small population size means that genetic drift influences their evolution. The isolated population undergoes rapid evolutionary change resulting in peripatric speciation.

4. No preservation: Because of their relatively small population size, the rapid pace of change, and isolated location, no fossils representing transitional forms of the new species happen to be preserved. Even if they had "gotten lucky" and fossilized, these fossils would have been at a different location from those of the parent population.

5. Reintroduction: Sea levels rise, reuniting the isolated snails with their sister lineage.

6. Expansion and stasis: The isolated population expands into its past range. Larger population size and a stable environment make evolutionary change less likely now. The formerly isolated branch of the snail lineage may outcompete their ancestral population, causing it to go extinct.
Fig. 5 A hypothetical example of punctuated equilibria. See text for explanation

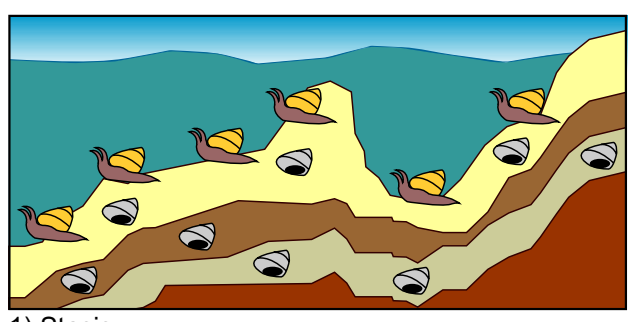

1) Stasis

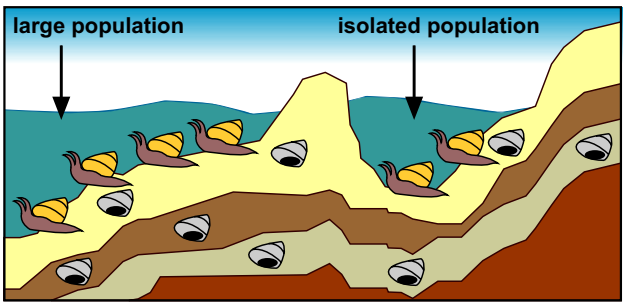

2) Isolation

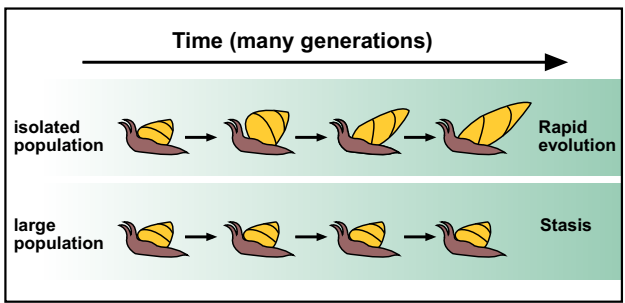

3) Strong selection and rapid change

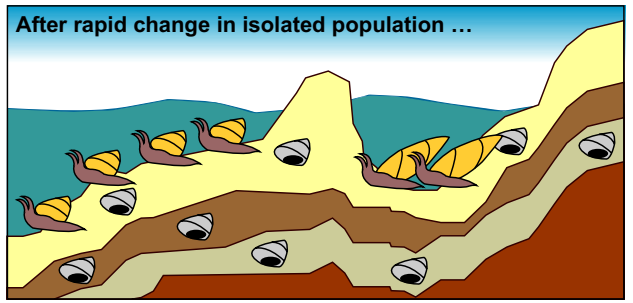

4) No preservation

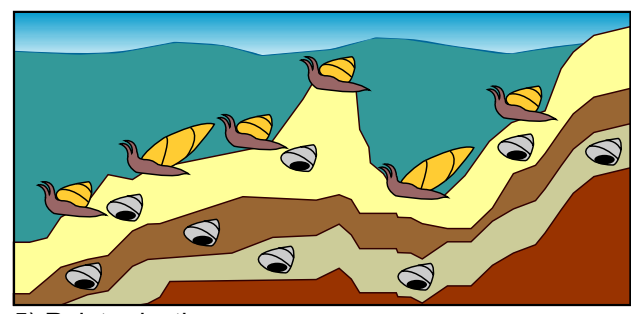

5) Reintroduction

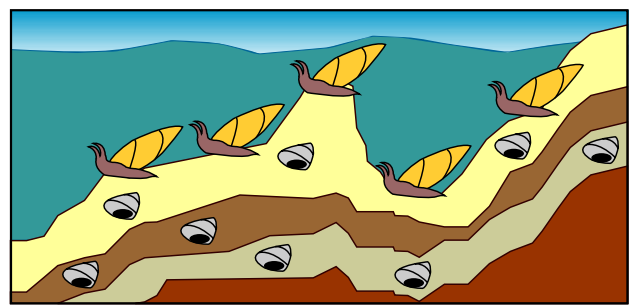

6) Expansion and stasis

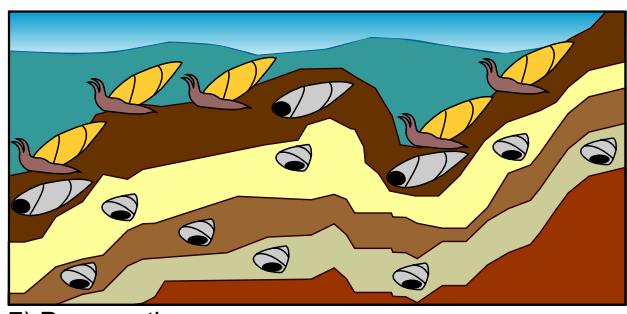

7) Preservation

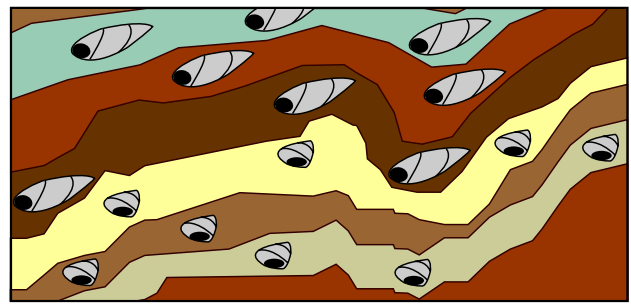

A punctuated pattern in the fossil record 
7. Preservation: Larger population size and a larger range move us back to step 1: stasis with occasional fossil preservation.

That, in a nutshell, is punctuated equilibria: a lot of evolutionary change occurring in a short period of time tied to a speciation event. Eldredge realized that this process would produce a punctuated pattern in the fossil record, in which one species seems to be replaced by a related species (Fig. 1a) or, if the new species does not outcompete the other, a pattern in which a new species "suddenly" appears (Fig. 1b).

There is nothing unusual about the evolutionary process proposed here. Speciation occurs at its normal rate, but it occurs in an isolated location. Evolutionary change only appears to be unusually "jumpy" after the fact because no transitional forms are preserved in the fossil record-but this is to be expected given the small size of the evolving population, its isolated location, and the nature of fossilization. Studies of modern organisms, like the apple maggot fly, have shown that the early stages of speciation can be observed in wild populations in just a few hundred years (Bush 1975). Fossilization, on the other hand, provides us with snapshots of biodiversity taken on much larger intervals - tens of thousands of years, if we are lucky - so it is not surprising that transitional forms are not always preserved.

\section{Alternative Explanations}

Of course, if we observe a punctuated pattern in the fossil record, punctuated equilibria are only one possible explanation. Scientists try hard to consider and investigate alternative explanations for what they observe. As an example, put yourself in the place of a paleontologist who observes the rock layers and fossils shown in Fig. 6. One species appears to have "suddenly" produced two daughter lineages, one anatomically identical to the ancestor, the other anatomically distinct. But appearances

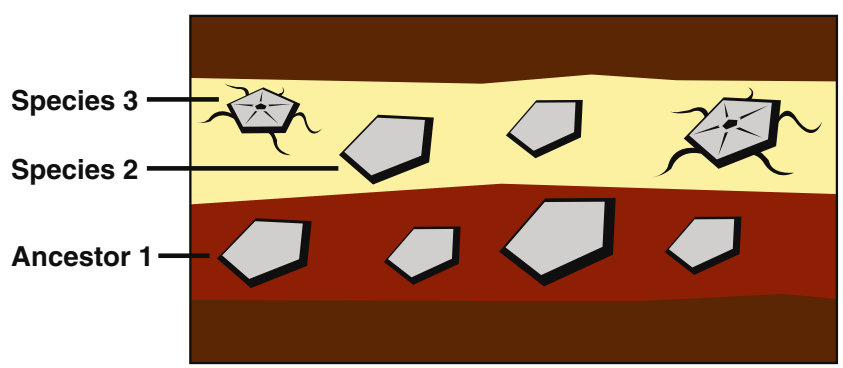

Fig. 6 A hypothetical stratigraphic record containing fossil echinoderms. Species 3 appears to have quickly evolved from Ancestor 1 can be deceiving. The fossils we have are only snapshots of actual history. What different scenarios could explain these snapshots? There are many possibilities, among them:

1. Punctuated equilibria-large amounts of change in a short period of time tied to a speciation event (Fig. 7a). Just a few hundred thousand years separate the two rock layers, and all of the evolutionary change connecting the ancestor to its descendents took place relatively rapidly - either in this location or nearby. Transitional forms between Ancestor 1 and Species 3 did exist, but they were not preserved in this part of the fossil record.

2. Phyletic gradualism-slow, steady divergence of lineages (Fig. 7b). Many millions of years separate the two rock layers. In this period of time, Species 3 gradually diverged from Ancestor 1 through a series of transitional forms, but these were not preserved because of poor conditions for fossilization.

3. Macromutation - a key mutation produces sudden evolutionary change skipping over transitional forms (Fig. 7c). Very little time separates the two rock layers, but that period encompasses a lot of evolutionary change. Species 3 was produced by a mutation that radically changed the offspring of Ancestor 1 in many ways; transitional forms did not exist. Such extreme mutants are sometimes called "hopeful monsters" or macromutations.

As each of these hypotheses is consistent with the fossils, to figure out which is most likely, we must look for other lines of evidence. Studies of genetics turn up no evidence that extreme yet adaptive macromutations of this sort occur, so macromutation seems an unlikely explanation. Punctuated equilibria and phyletic gradualism, on the other hand, are both genetically plausible. However, they lead us to expect that different amounts of time separate the two rock layers. If stratigraphic and isotopic dating studies were to point to a short interval, the data would support punctuated equilibria; a long interval would be consistent with phyletic gradualism.

\section{Punctuated Equilibria and Parsimony}

The idea of punctuated equilibria is an important, but oftenmisinterpreted, model of how evolutionary change happens. The model does not:

- Suggest that Darwin's theory of evolution by natural selection is wrong

- Contradict the central ideas of evolutionary theory, that life is old and organisms share common ancestors 
Fig. 7 Three plausible evolutionary scenarios that explain the stratigraphic record shown in Fig. 6. (a) Species 3 diverges from Ancestor 1 in a manner consistent with punctuated equilibria. (b) Species 3 diverges from Ancestor 1 very gradually, but intermediate forms are not fossilized. (c) Species 3 diverges from Ancestor 1 via

macromutation
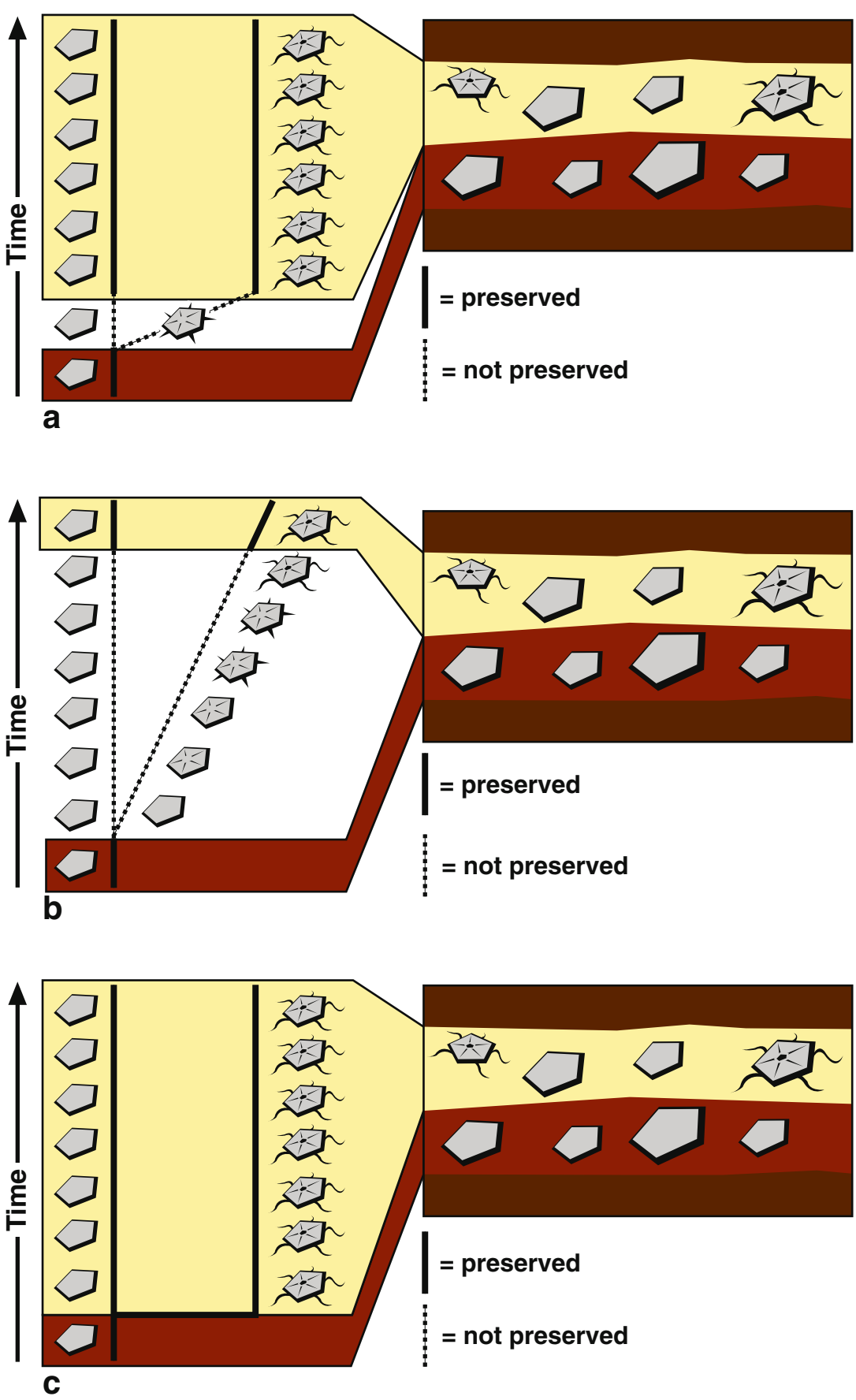

- Negate previous work on how evolution by natural selection works

- Imply that evolution only happens in rapid bursts

It simply suggests that established mechanisms of evolutionary change (speciation, migration, natural selection, and genetic drift) might work together to help explain a formerly mysterious pattern seen in the fossil record. In this way, Eldredge and Gould's proposal embodies a key criterion by which all of science operates: parsimony.
The principle of parsimony suggests that when two explanations fit the observations equally well, the simpler explanation - in this case, the one that does not involve proposing an entirely new mechanism of evolution-should be preferred over a more convoluted and complex explanation. After all, why propose a new mechanism when the old ones (which are already supported by many lines of evidence) work perfectly well to explain what we observe?

In science, theories are rejected and radical changes occur when existing ideas cannot account for the available 
evidence as well as a new idea. Some antievolution groups suggest that evolutionary theory finds itself in this precarious situation-but that is a misrepresentation. In fact, all the available evidence (from biology, geology, and even chemistry and physics) is consistent with evolution. Even punctuated patterns in the fossil record fit well with our understanding of how evolution works. The punctuated equilibria model is not a challenge to evolution, but is rather a deeply connected node in the web of ideas that forms this theory.

\section{Give Me an Example of That}

Want more examples of the principle of parsimony in action? Check these out:

- Parsimony applied to phylogenetics. The principle of parsimony has applications in all of science-from paleontology to particle physics. When known processes can explain the available evidence, we have no reason to propose a new, more complex explanation. Here, we have seen that principle applied to patterns in the fossil record, but the same reasoning applies equally well in other areas of evolutionary biologyparticularly in the field that reconstructs evolutionary relationships among organisms: phylogenetics. Find out how biologists use parsimony to build evolutionary trees: http://evolution.berkeley.edu/evolibrary/ article/usingparsimony_01

- Using trees to understand plants. Just as Niles Eldredge observed patterns in the fossil record and came up with a parsimonious explanation for them, other biologists observe patterns in living organisms and try to come up with similarly parsimonious explanations for those patterns. This research profile follows scientist Chelsea Specht as she pieces together the evolutionary history of tropical plants and their pollinators and applies the principle of parsimony to the problem: http://evolution.berkeley. edu/evolibrary/article/specht_01

\section{Branch Out}

Explore ideas related to the concepts described here.

- Punctuated equilibria help explain some sorts of patterns that we can observe in the fossil record-but there is more to be learned from studying these patterns more broadly. Through detailed analysis of the fossil record, scientist David Jablonski reconstructs the rules that helped dictate who lived and died in past mass extinctions. This research profile describes his surprising discoveries and their disturbing implications for the biodiversity crisis today. Learn more online: http:// evolution.berkeley.edu/evolibrary/article/jablonski_01

- The process of punctuated equilibria is based on the ideas of allopatric and peripatric speciation; however, biologists think that speciation can happen even when subpopulations are not isolated geographically. Review speciation by geographic isolation and get an introduction to parapatric and sympatric speciation: http://evolution.berkeley.edu/evolibrary/article/ speciationmodes_01

\section{Dig Deeper}

Visit Understanding Evolution online to find out even more about some of the concepts addressed here.

- The history of the concept of allopatric speciation: http:// evolution.berkeley.edu/evolibrary/article/history_21

\section{In the Classroom}

One might imagine punctuated equilibria to be a topic appropriate only for biology majors-but, in fact, the subject has something to offer students at many levels. It relies on many basic concepts in evolution and the nature of science, and, because the topic is often misrepresented by antievolution groups as a challenge to evolutionary theory, it can provide a context for discussing the nature of scientific theories and scientific controversy. Most high school students are capable of tackling punctuated equilibria directly. You might introduce the idea of punctuated patterns in the fossil record with this lesson:

- Fossil Patterns: Gradualism vs. Punctuated Equilibria, from the Evolution and the Nature of Science Institutes. In this activity for grades 9-12, students learn the differences between gradualism and punctuated equilibria by manipulating two sets of simulated fossils (Caminalcules). http://www.indiana.edu/ ensiweb/ lessons/peek.html

You can also help prepare students at lower grade levels for this topic by helping them understand the process of fossilization, how scientists make inferences based on the fossil record, and how scientists develop and investigate 
hypotheses about events in the past - concepts addressed by these lessons:

- Getting into the Fossil Record, from the UC Museum of Paleontology. In this interactive module for grades 68 , students are introduced to fossils and the fossilization process by examining how fossils are formed and the factors that promote or prevent fossilization. http:// www.ucmp.berkeley.edu/education/explorations/tours/ fossil/index.html

- Stories from the Fossil Record, from the UC Museum of Paleontology. This web-based module for grades 612 provides students with a basic understanding of how fossils can be used to interpret the past. http://www. ucmp.berkeley.edu/education/explorations/tours/stories/ index.html

- Sequencing Events, from Modeling for Understanding in Science Education. In this activity for grades 9-12, students attempt to sequence and create a story around a series of cartoon frames to serve as the basis for a discussion about how decisions are made and how arguments are constructed in science. http://www.wcer. wisc.edu/ncisla/muse/naturalselection/materials/ section $1 /$ lesson1B/index.html
At the undergraduate level, students can even dig into the original research papers that developed the idea of punctuated equilibria:

- Eldredge and Gould (1972) http://www.nileseldredge. com/pdf_files/Punctuated_Equilibria_Eldredge_Gould 1972.pdf

- Gould and Eldredge (1977) http://www.nileseldredge. com/pdf_files/Punctuated_Equilibria_Gould_Eldredge 1977.pdf

\section{References}

Bush GL. Sympatric speciation in phytophagous parasitic insects. In: Price PW, editor. Evolutionary strategies of parasitic insects and mites. New York: Plenum; 1975. pp. 187-206.

Eldredge N. Editor's corner: The early "evolution" of "punctuated equilibria." Evolution: Education and Outreach 2008. DOI 10.1007/s12052-008-0032-0.

Eldredge N, Gould SJ. Punctuated equilibria: An alternative to phyletic gradualism. In: Schopf TJM, editor. Models in paleobiology. San Francisco: Freeman, Cooper; 1972. pp. 82-115.

Gould SJ, Eldredge N. Punctuated equilibrium comes of age. Nature 1993;366:223-7. 